\title{
Measuring Level-K Reasoning, Satisficing, and Human Error in Game-Play Data
}

\author{
Tamal T. Biswas \\ Department of CSE \\ University at Buffalo \\ Amherst, NY 14260 USA \\ Email: tamaltan@buffalo.edu
}

\author{
Kenneth W. Regan \\ Department of CSE \\ University at Buffalo \\ Amherst, NY 14260 USA \\ Email: regan@buffalo.edu
}

\begin{abstract}
Inferences about structured patterns in human decision making have been drawn from medium-scale simulated competitions with human subjects. The concepts analyzed in these studies include level- $k$ thinking, satisficing, and other human error tendencies. These concepts can be mapped via a natural depth of search metric into the domain of chess, where copious data is available from hundreds of thousands of games by players of a wide range of precisely known skill levels in real competitions. The games are analyzed by strong chess programs to produce authoritative utility values for move decision options by progressive deepening of search. Our experiments show a significant relationship between the formulations of level-k thinking and the skill level of players. Notably, the players are distinguished solely on moves where they erred-according to the average depth level at which their errors are exposed by the authoritative analysis. Our results also indicate that the decisions are often independent of tail assumptions on higher-order beliefs. Further, we observe changes in this relationship in different contexts, such as minimal versus acute time pressure. We try to relate satisficing to insufficient level of reasoning and answer numerically the question, why do humans blunder?
\end{abstract}

Key words. Game theory, level-k thinking, blunder, satisficing.

\section{INTRODUCTION}

Decision making under risk and uncertainty is a prime focus of several disciplines in mathematical and social sciences, ranging from mathematical decision theory through machine learning and artificial intelligence to economics and psychology. The path of entry to the former emphasizes normative utility values and costs, competitive strategies, theoretical equilibria, and descriptive theories of behavior. The AI and human-behavior sides start from how knowledge is gained and search may be strategized. The latter areas include concepts of behaviors that deviate from rationality and theoretically optimal (equilibrium) strategies. Studies applying these approaches have involved staged competitions with human subjects dedicated to the study; we mention in particular the "Iowa gambling study" [1], [2], the "Colonel Blotto game" [3], the "11-20 money request game" [4], [5], the "beauty contest" game as implemented by [6], and others in [7], [8], [9]. Their concepts include $k$-level reasoning [10], [11], which involves estimating the depth of interaction with one's opponent(s), satisficing introduced by [12], which means "settling" for a known outcome without looking deeper for a better one, and other forms of human (or agent) fallibility based either on insufficient resources for managing complexity (bounded rationality) or on lapses of attention or effort. For example, the 11-20 game enables each player to select a prize of any value from $\$ 11$ to $\$ 20$, with a $\$ 20$ bonus if the value is exactly $\$ 1$ less than the opponent's value. A satisficer might just take $\$ 20$ and run, whereas a level-1 strategizer might try $\$ 19$, a level-2 thinker $\$ 18$, and so on.

The distinctive approach in this paper is to map these concepts into a long-established game domain, namely competitive chess, in which large amounts of data from real competitions are available from millions of moves played in hundreds of thousands of high-level recorded games. The decisions-indeed the utilities of all alternative moves besides the one chosen - are authoritatively judged by chess programs run to high search depths at which they outperform any human player. The common element of depth in the human cognitive concepts maps to the progressively increasing search depths, at each of which the programs report values for each move. The manner in which these values change between depths-as deeper flaws in "trap" moves and deeper value in "surprise" moves are revealed-affords precise mathematical formulations of the mapped concepts. Thus we blend the mathematical and psychological approaches at an instrumental level below the top-level analysis of our experiments. The goal is to test how well conclusions from these studies hold up under this mapping into a rich-data environment.

Our most distinctive element over many previous chessbased studies [13], [14], [15], [16], [17], [18], [19], [20], [21], [22], [23] is the employment of chess program values at lower depths of search. This seems quixotic insofar as the highest-available depth values are the most authoritative, and any change in value of a move could be regarded as correcting a flaw in less-intensive data-taking. The use of depth by [24], [25] involves only the top two depths. Following [26] we use the intermediate-depth utility values as markers for the gain and taking stock of knowledge. Via these markers we give mathematical formulations for patterns that underpin concepts of level- $k$ reasoning and sensitivity to changes in information or extrinsic value of options. We seek deeper roots for behaviors that have not been convincingly captured by a single-thread approach, notably the ability to handle 
complexity and change, satisficing behavior, and the human tendency to blunder.

We highlight a particular kind of mistaken decision: one that looks best at first sight but whose drawbacks are revealed by deeper consideration. Satisficing-Herbert Simon's portmanteau of "satisfy" and "suffice"-is recognized by [27] as "as a decision-making strategy or cognitive heuristic by which humans search through available alternatives until an acceptability threshold is met, whereupon they stop searching and choose.' We seek its origin in a middle ground between two modes of thought highlighted by Daniel Kahneman and Amos Tversky and others in various studies [28], [29], [30], [31], [32], [33], [34]: thoughts and preferences that come naturally to mind without reflection, versus a controlled but slow mode of deliberate thinking. It is easy to find conditions for blundering in the former, but we believe that progress on analyzing patterns that lead to blunders will come via the following questions:

- How can we treat satisficing in terms of the above quantified criteria?

- How can we measure the level- $k$ thinking threshold for satisficing?

- How deeply must an expert decision maker in any particular think to be reasonably sure of a good outcome?

- How much difference between decision makers at various depth levels should be expected in order to frame a good group decision?

We report progress from chess experiments mainly on the first two questions. We define a metric of swing in value across depths that yields a precise formulation of a depth threshold for satisficing. We demonstrate that the measured threshold varies uniformly with a player's rated skill. It is notable that we obtain a strong relationship while considering only those moves on which strong players made mistakes - as opposed to the many other moves on which they demonstrate their skill positively. Thus we have isolated a sub-surface phenomenon that affects human decision making under adversarial conditions of imperfect knowledge. We investigate this also in games under greater time pressure.

Our approach is different from usual level- $k$ models where the population is partitioned into types that differ in their depth of reasoning. A player of level- $k$ type takes action considering the opponent is a level $k-1$ type. Such notions are clear in the "11-20" game defined above where the type is identified simply by the number chosen. The above-cited papers study how these types change as players "learn" in repeated plays of the game, but this setting promotes unidirectional results. The real-world difference here is that " $k$-ness" is fluid with players engaged in hundreds of repetitions of the basic move-choice task in variegated game positions under budget constraints of concentration energy and thinking time. The use of values from all program search depths affords modeling players as distributions over thinking at various levels " $k$." Thus we provide a large-data refracting prism by which to isolate robust conclusions across a spectrum of situations and skill levels.

\section{Chess Data And Methodology}

Humans and computers are both rated on the common Elo scale [35] on which 1000 is a typical rating for a novice player, 1600 for an avid amateur player, 2200 is considered "master," and only the past (retired) world champion Garry Kasparov and the current world champion Magnus Carlsen have ever achieved ratings considerably beyond 2800 . The computer programs Stockfish 6 and Komodo 9 are however rated above 3300 on common PC hardware, and many other programs and versions surpass 3200 [36]. A 400-point advantage in rating confers over $90 \%$ expectation in matches and is considered total superiority. In the meantime, chess retains extraordinary absolute complexity with game trees of average depth 80 plies (meaning a move for White or Black) and average branching factor (i.e., number of legal moves in a position) about 35 [37]. This combination is distinctive to chess as opposed to Go [38].

Iterative deepening means that the search in a given position $p$ is organized in rounds of basic depth $d=1,2,3, \ldots$ plies. Via the standard Universal Chess Interface (UCI) protocol, the engine reports values $v_{i, d}$ for every legal move $m_{i}$ in $p$ at every depth $d$. The search ends at a maximum depth $D$ that is either fixed or determined by the engine's own time budgeting. Stockfish reports values from $d=1$ but can reach depths $D$ above 20 within minutes on common PC hardware.

All the programs give similar values in relation to the win expectation curve [39], which is a smooth logistic curve

$$
P(\theta)=\frac{1}{1+e^{-a \theta}}
$$

where $\theta$ and $a$ are the evaluation of a position and an engine dependent variable respectively. As reported in Figure 1, the $a$-values of $a$ for the engines are close.

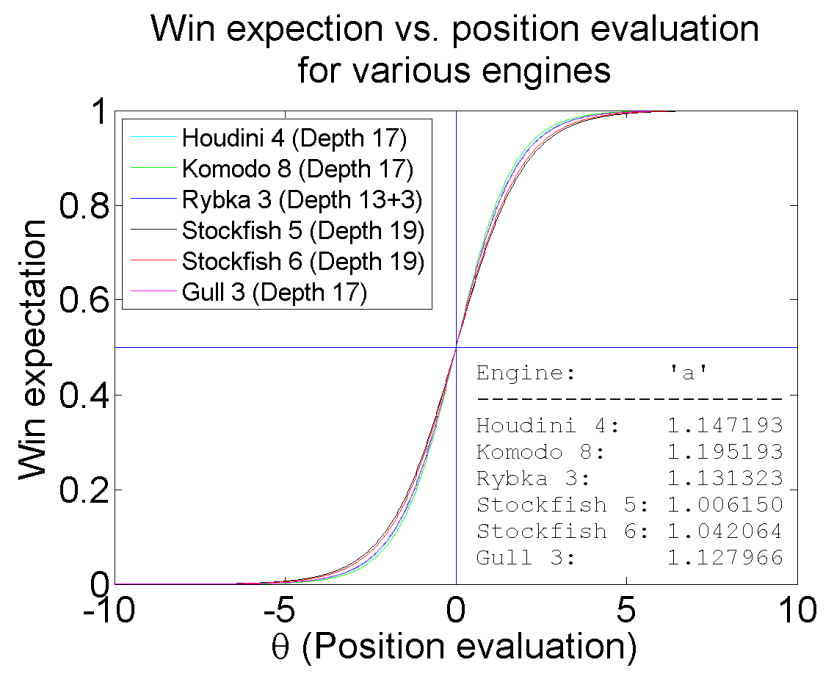

Fig. 1. Win expectation vs. position evaluation for various engines

Table I demonstrates an example of the data obtained by setting the engine in "Multi-PV" mode to give equal consideration to all legal moves (a cap of 50 served), with 
TABLE I

EXAMPLE DATA FROM STOCKFISH 6. BLACK TO MOVE (POSITION CODE 8/7B/8/2PKP2R/P6P/PBK5/6PP/3R4 B), SO LOWER IS BETTER

\begin{tabular}{|l|l|l|l|l|l|l|l|l|l|l|l|l|l|l|l|l|l|l|l|}
\hline & 1 & 2 & 3 & 4 & 5 & 6 & 7 & 8 & 9 & 10 & 11 & 12 & 13 & 14 & 15 & 16 & 17 & 18 & 19 \\
\hline Ra1 & 187 & 187 & 187 & 187 & 206 & 197 & 209 & 075 & 027 & 021 & 041 & 012 & 017 & 010 & 006 & 006 & 005 & 005 & 005 \\
\hline Re1 & 175 & 198 & 173 & 132 & 126 & 136 & 092 & 129 & 088 & 074 & 082 & 059 & 053 & 062 & 065 & 055 & 045 & 031 & 040 \\
\hline h3 & 182 & 182 & 092 & 225 & 118 & 118 & 098 & 103 & 090 & 082 & 078 & 054 & 048 & 043 & 082 & 088 & 086 & 070 & 069 \\
\hline Kc6 & 192 & 192 & 192 & 198 & 189 & 174 & 189 & 180 & 126 & 114 & 129 & 129 & 132 & 105 & 089 & 089 & 093 & 100 & 106 \\
\hline Rg1 & 184 & 184 & 184 & 105 & 095 & 108 & 098 & 129 & 111 & 100 & 110 & 127 & 131 & 131 & 138 & 117 & 126 & 125 & 119 \\
\hline Rc1 & 053 & 166 & 174 & 191 & 108 & 122 & 095 & 150 & 078 & 098 & 110 & 107 & 113 & 113 & 094 & 093 & 089 & 115 & 121 \\
\hline
\end{tabular}

evaluations from white's point of view in units of centipawns, figuratively hundredths of the value of a pawn. The moves are sorted by their evaluation at the highest depth. The grid reveals that the move "Rc1" was the best candidate at the beginning of the search process, but was superseded by the ultimate best move "Ra1" from depth $\mathbf{8}$ onward. We can say that its inferiority was exposed between depths $\mathbf{7}$ and $\mathbf{8}$, and this underlies our notion of depth of satisficing.

In symbolic terms, we represent the moves as $m_{i}$ where $i \in(1 \cdots \ell)$ and $\ell$ is the number of legal moves. The move $m_{1}$ represents the engine provided best move at depth $D$, the highest depth of analysis. Each value $v_{i, d}$ in the table is the evaluation of move $m_{i}$ at depth $d$, while the best evaluation at depth $d$ is denoted by $v_{*, d}$. These values are scaled as described and justified in [20], [21], [26]; the details do not concern us here. The difference in optimality of $m_{i}$ at depth $d$ is denoted by

$$
\delta_{i, d}=v_{*, d}-v_{i, d},
$$

The best move provided by the engine at the highest depth has $\delta_{1, D}=0$, while simple blunders are characterized by high $\delta_{i, d}$ values across all or many depths. We identify two other main categories of moves, swing-up and swing-down moves, as follows:

- A move swings up if its delta values with respect to the best move at any depth decrease with increasing depth. Figuratively, these are moves whose potential is only manifested at higher depth.

- A move swings down if an initially low delta value becomes markedly higher with increasing depth, indicating it is ultimately a poor move to play.

The definition of swing for move $m_{i}$ is the sum of differences in delta values between any depth $d$ and the highest depth $D$ :

$$
s w\left(m_{i}\right)=\sum_{d=1}^{D}\left(\delta_{i, d}-\delta_{i, D}\right) .
$$

For a swing-up or swing-down move the value $s w\left(m_{i}\right)$ is positive or negative respectively. Note that it is possible for an inferior move to have positive swing - it may be less inferior than it was at earlier depths.

Finally, for any move $m_{i}$ other than $m_{1}$ that was ranked higher than $m_{1}$ at some earlier depth, we can define $d_{i}$ to be the maximum $d$ such that $v_{i, d}>v_{1, d}$. The swing measure $s w\left(m_{i}\right)$ quantifies how attractive $m_{i}$ was at earlier stages of consideration relative to its ultimate inferiority. It can serve as a weight on $d_{i}$ or a selection criterion for $d_{i}$; our further measures of "difficulty" and "complexity" in [26] are analogous to the former but here we do only the latter. The average of $d_{i}$ for moves $m_{i}$ in a sample-selected by a threshold on $s w\left(m_{i}\right)$ and characteristics of the player(s) involved-is the depth of satisficing in the sample.

Our main data set comprised games where both the players are within 10 Elo rating points of a milepost value 2200, $2300, \ldots, 2700$. Each of the games was run under standard time controls, typified by 90 minutes for the first 40 moves followed by 30 minutes for the rest of the game with an additional 30 seconds per move after the 60th move. Our second data set comprises 589 games containing 42,753 moves in tournaments since 2007 where the current and previous world champions both participated. These games featured the world's elite players and give a general overview of the style of game-play and positions these players face and produce. The tables presented here come from analysis by Stockfish 6; we obtained closely similar results using Komodo 9. Both engines were configured to produce analysis up to depth 19 producing up to 64 principal variations at each depth. We have analyzed these recorded evaluations to quantify depth for satisficing and estimate player quality.

\section{RESUlTS}

Table II shows the distribution of swing-up (where played move has positive swing) versus swing-down (where played move has negative swing) moves. This data represents that with increasing skill, players play fewer negative swing moves and overall for any rated players, the number of swing down moves is below $20 \%$ of the total moves.

TABLE II

MiLEPost DATA SET STATISTICS

\begin{tabular}{|l|c|c|c|c|c|}
\hline Dataset & \#moves & Swing+ & Swing- & Swing+ \% & Swing- \% \\
\hline RR2200 & 35893 & 29769 & 6124 & $82.94 \%$ & $17.062 \%$ \\
\hline RR2300 & 41757 & 34943 & 6814 & $83.68 \%$ & $16.32 \%$ \\
\hline RR2400 & 37768 & 32099 & 5669 & $84.99 \%$ & $15.01 \%$ \\
\hline RR2500 & 44185 & 38818 & 5367 & $87.85 \%$ & $12.15 \%$ \\
\hline RR2600 & 67772 & 59115 & 8657 & $87.23 \%$ & $12.77 \%$ \\
\hline RR2700 & 22414 & 19757 & 2657 & $88.15 \%$ & $11.85 \%$ \\
\hline
\end{tabular}

Figure 2 demonstrates the average error for both the played move and the engine move versus depth of analysis for all moves without any distinction. Figure 2a shows the results for 2200 Elo players, whereas Figure 2b shows results for 2700 Elo players. We observe no significant distinction in the 


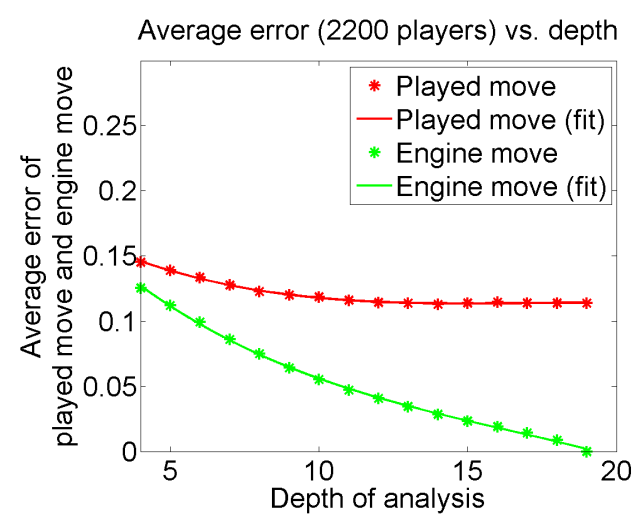

(a) 2200 Players

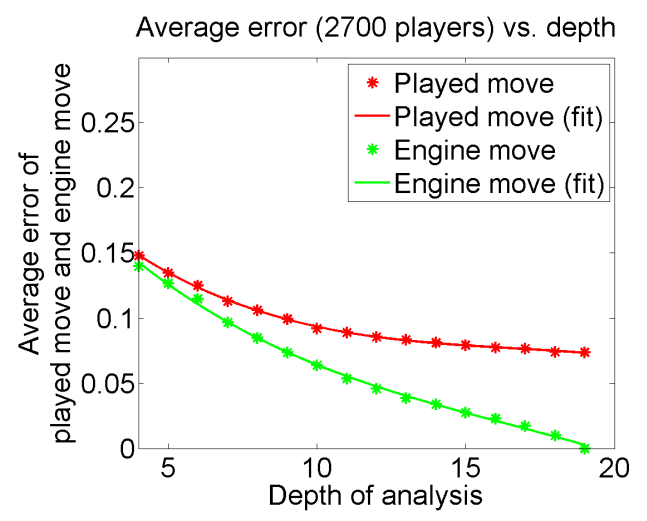

(b) 2700 Players

Fig. 2. Average aggregate error for played move and engine move vs. depth for all moves

playing pattern of various levels of skilled player with respect to depth. All positions can be divided into two groups:

- Played move having negative swing ("swing down");

- Played move having positive swing or zero ("swing up").

Figures $3 \mathrm{a}$ and $3 \mathrm{~b}$ again show little difference between rating level when considering only swing-up moves. Though the graph demonstrates that the played-move swing for higherrated players matches the engine move swing much more closely, still no distinction between various depths is prominent.

Finally, we consider the set of positions with played moves with negative swing. Figure 4 graphs the average error for the played move and the engine move versus depth for 2200 and 2700 players. The played-move curves for players between these ratings (not shown) fall in between those of these plots. Upon fitting all the curves by cubic polynomials, for each and every plot we find a very distinct intersection point where the engine move error and played move error intersect. This is a persistent empirical phenomenon. It is indicated already by the relative nearness of the curves at low depths over all moves and the steady apartness at low depths over swing-up moves. We call the depth at this intersection point the aggregate depth of satisficing for the sample.

Graphing it versus rating clearly shows how the aggregate depth of satisficing moves towards higher depth with increas-

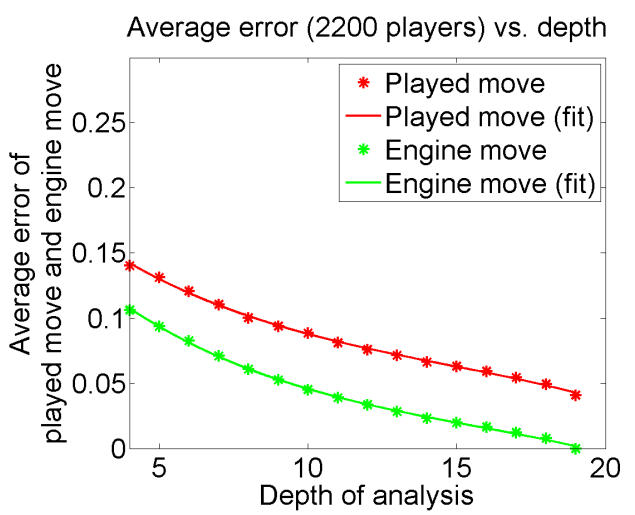

(a) 2200 Players

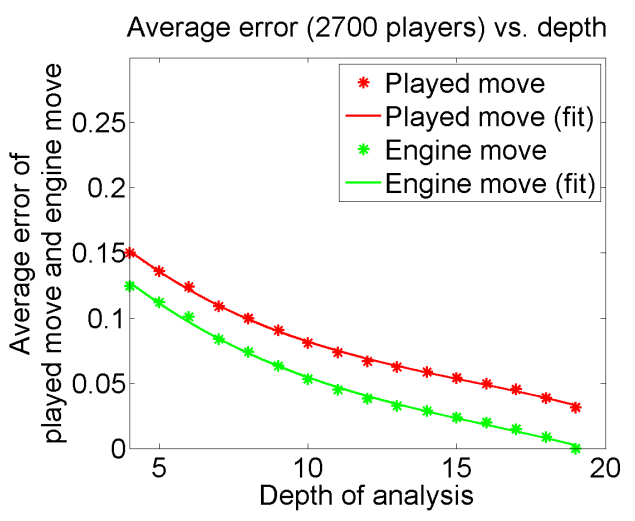

(b) 2700 Players

Fig. 3. Average aggregate error for played move and engine move vs. depth for moves with positive swing

ing skill. The depth at this intersection point is where the engine move and played move are reversing their role, i.e., before this point, the played move had lower delta at that particular depth than the engine move. Beyond the depth of satisficing, the played move's evaluation becomes relatively worse. We say a strong player goes deeper before being tricked, whereas a lower-skilled player gets tricked easily without further contemplation.

Thus swing-down moves, although comprising less than $20 \%$ of the total moves, provide a clear indication of the player's skill level and show a decision maker's threshold of blundering. This small number of turns also contain more information to distinguish the player than turns where the player agreed with the engine's first move and those with played move swinging up. The intersecting points clearly define how players of different skill levels can be segregated best on the depth of satisficing.

Next we analyze these moves further to figure out whether this phenomenon is consistent across the whole game and how it is affected by time pressure on decision making. Again, owing to the standard time control at 40 moves, we expect to see the effect of time pressure at turns closer to 40 . Even toplevel players use most of their time before turn 30 and are then forced to play without much thinking time until the refresh at move 41. Similar conditions apply to the ends of long games. 


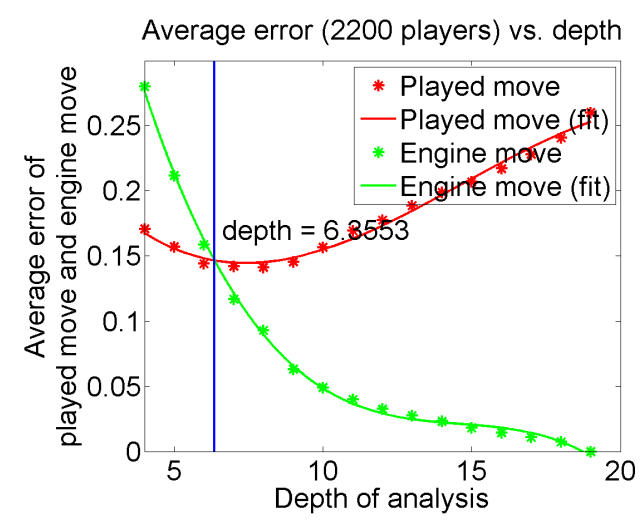

(a) 2200 Players

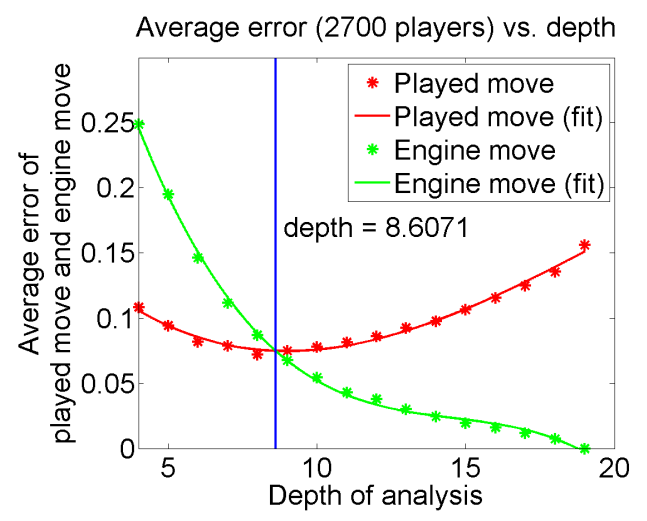

(b) 2700 Players

Fig. 4. Average error for played move and engine move vs. depth for moves with negative swing

Our analysis shows a significant effect that is ascribable to this behavior. We have considered all swing-down played moves before turn 40 . We split this selection in almost two equal parts, one containing moves numbered 9 to 25 , the other from 26 to 40 . Figure 5 shows how 2200 and 2700 rated players played at the beginning of the game play from move 9 to move 25 . We find, the depth of satisfying is higher for 2700 players than 2200 players. But for both these players the depth for satisficing is higher than that we achieved when we considered all moves. This phenomenon is consistent for every milepost rating level.

Figure 6 shows 2200 and 2700 players' depth for satisficing in time pressure. As expected, both the players play subpar in time pressure as the depth for satisficing gets reduced drastically for moves between 26 and 40. In figure 7, we have plotted the depth for satisficing for all these three scenarios over players of all the ranks. A simple linear fit nicely represents the relation between the skill and the depth of satisficing. In the next section we further demonstrate how far this relationship holds for individual players.

\section{Case Study on Quality and Blunders}

Our final data set comprises games from recent top-level events involving the previous and present world champions, V. Anand and M. Carlsen. Selecting the games between them,

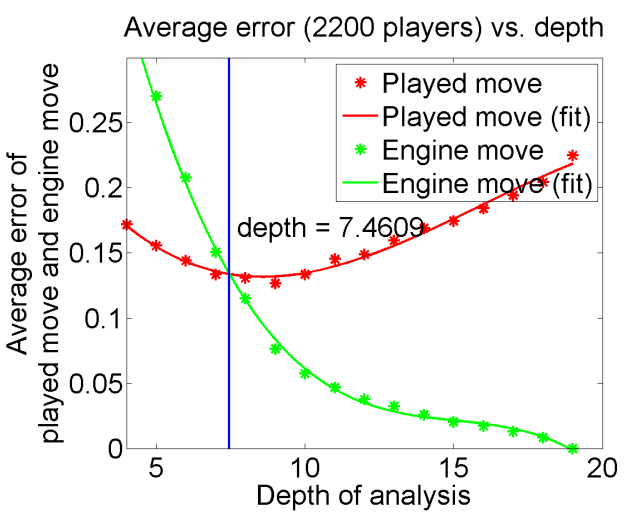

(a) 2200 Players

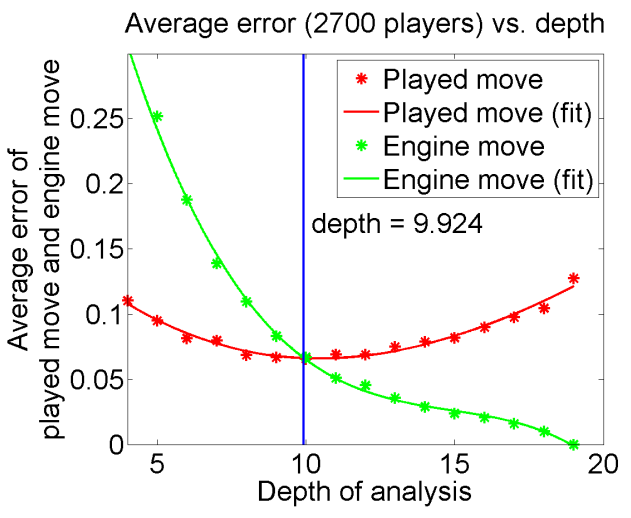

(b) 2700 Players

Fig. 5. Average error for played move and engine move vs. depth for moves between 9 and 25 with negative swing

we try to investigate the effect of the depth for satisficing for these two players at different phases of the game. We further try to relate their strength of play at those positions by their swing down played moves.

We derive a mapping from the depth for satisficing to the Elo for three scenarios namely, all moves, moves from 9 to 25 and moves from 25 to 40 . From the linear fit presented in Figure 7, we come up with the conversion as follows:

$$
\begin{aligned}
& E l o_{a}=\left(S_{a}+3.5521\right) / 0.0045 \\
& \text { Elo }_{b}=\left(S_{b}+3.7034\right) / 0.0050 \\
& \text { Elo }_{c}=\left(S_{c}+6.5687\right) / 0.0054
\end{aligned}
$$

where $E l o_{a}, E l o_{b}, E l o_{c}$ represents Elo considering all moves, till moves 25, and for moves 26-40 respectively. Similarly, $S_{a}, S_{b}, S_{c}$ represents the respective depth for satisficing.

For an extremal test we apply this scale to the Anand and Carlsen as shown in Figure 8 and the following table.

TABLE III

ESTIMATION OF ELO

\begin{tabular}{|l|l|l|l|l|l|l|}
\hline & \multicolumn{2}{|l|}{ All Moves } & \multicolumn{2}{l|}{ Moves 9-25 } & \multicolumn{2}{c|}{ Moves 26-40 } \\
\hline & $S_{a}$ & $E l o_{a}$ & $S_{b}$ & $E l o_{b}$ & $S_{c}$ & $E l o_{c}$ \\
\hline M. Carlsen & 9.348 & 2867 & 11.095 & 2960 & 8.5238 & 2794 \\
\hline V. Anand & 9.8407 & 2976 & 10.885 & 2918 & 9.3472 & 2947 \\
\hline
\end{tabular}




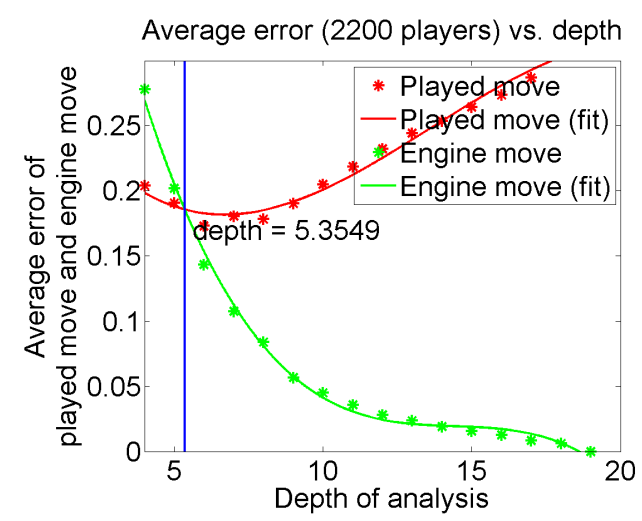

(a) 2200 Players

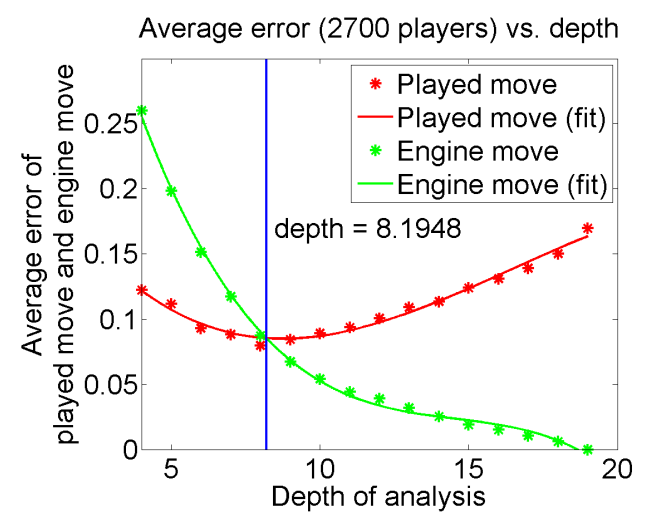

(b) 2700 Players

Fig. 6. Average error for played move and engine move vs. depth for timepressure moves between 26 and 40 with negative swing

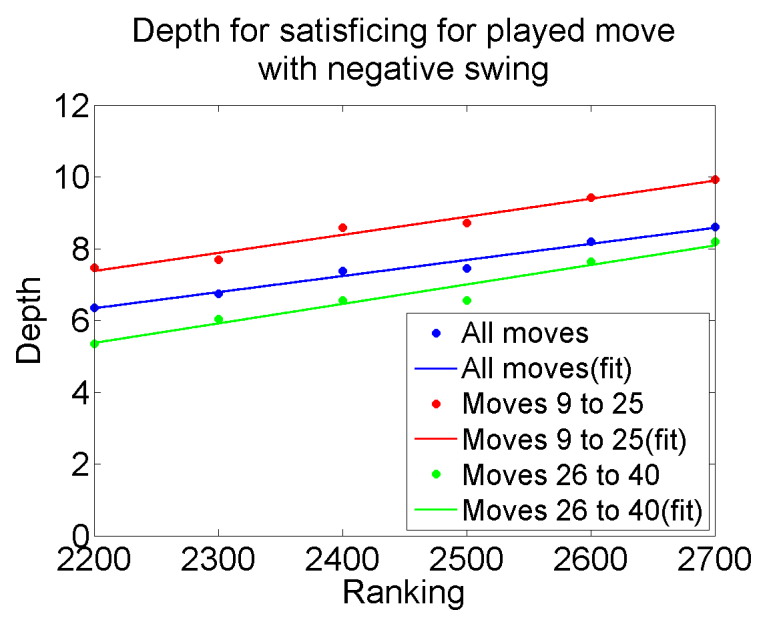

Fig. 7. Depth of satisficing

Table III infers that Carlsen considerably thought at a higher depth at the beginning of chess games, which might explain his observed propensity to outplay other players immediately after the early opening phase and the secret for his very high Elo for blitz and rapid games. The model reports ratings for Anand that are higher than his actual Elo. That signifies a lower tendency to blunder due to the insignificant from insufficient depth of reasoning. Considering all moves, we found Anand

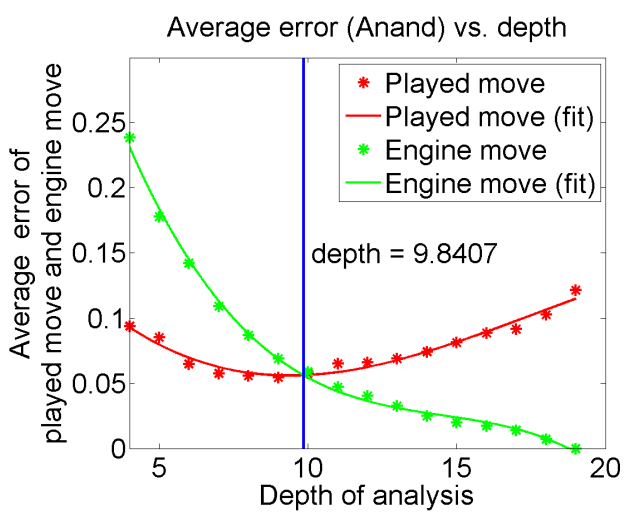

(a) Anand

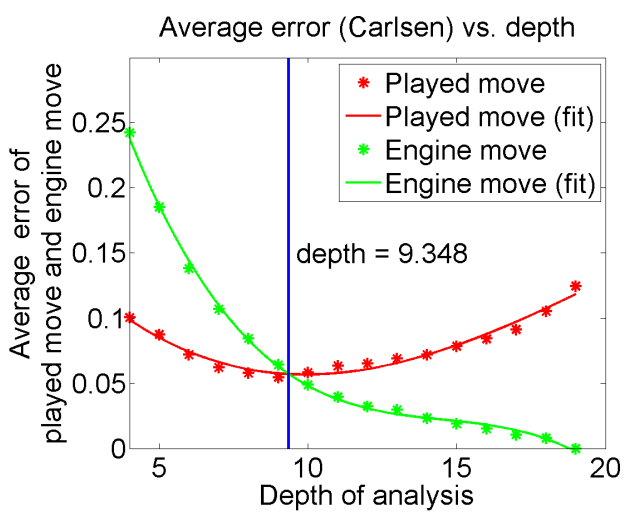

(b) Carlsen

Fig. 8. Depth for satisficing for Anand and Carlsen

makes 3.63\% more overall error compared to Carlsen, inferred as lower ability to find the optimal move when the played move and engine move both swing up.

\section{CONClusions ANd Future Work}

We have quantified measures for level- $k$ thinking and satisficing in chess and have demonstrated their impact from realworld data. We have coined and justified a new term 'depth for satisficing' to quantify the measure of 'satisficing' and found a strong correlation between skill level and depth for satisficing. It is noteworthy that the results were derived from selecting moves representing mistakes by strong players, and via lowerdepth numeric components that in analogous situations might have been thought dismissible as "noise." We show that these errors contain much information about the decision maker in a most concise way.

The strength of our correlation lends support to the interpretation that the players go with the highest-level estimations of value that they reach, disregarding considerations at earlier stages. In the analogous situation of the 11-20 game, the Nash-optimal strategy is a distribution over the ten number choices that includes playing the "naive" choice of '20' exactly $5 \%$ of the time, down to $25 \%$ for ' 15 ' and nothing below, which [5] shows is approached in time pressure but not under repeated plays. In the chess setting, similar use of a distribution would convey epistemologically doubting one's conclusions 
and giving weight to earlier estimates in what would be felt as a roll of the dice or intuitive leap. Our results speak that were such behavior in force in high-level chess at standard time controls, then we would see a fuzzier, noisier correlation with significantly lower overall depth values — such as we do see for time-pressure moves. Thus we regard our large field data as supporting the reality of non-equilibrium behavior under the conviction of the estimation reached at a "plateau" of the thinking process, and further, Strzalecki's contention [9] that the level- $k$ choices are largely "independent of the tail assumptions on the higher order beliefs."

Our model is conducive to formulating the difference between a blunder and a gamble. The latter means an objectively sub-optimal move that nevertheless poses such a high challenge to the opponent that the likelihood of an opponent's error gives the player a higher expectation than the optimal (i.e., "safest") move. This requires identifying game decisions where players make a sub-optimal decision with the divinable intention to decoy the opponent. Another aspect to investigate is error recovery, which may follow unsuccessful gambling or inadequate long-term planning, and might profit from a blunder by the opponent. A longer objective is a learning model for humans that generates problems for humans, provides feedback based on the responses, and tailors the difficulty and complexity of subsequent problems accordingly in order to aid in the learning process of the human. We hope that if artificial agents can be produced for other decision domains, this same approach can be applied to judge human decisions more accurately [40], replacing reactive criticism of mistakes by considerations of depth as a measure of expertise.

\section{REFERENCES}

[1] A. Bechara, A. Damásio, A. Damásio, and H. Anderson, "Insensitivity to future consequences following damage to human prefrontal cortex," Cognition, vol. 50, pp. 1-3, 1994.

[2] A. Bechara, H. Damasio, D. Tranel, and A. Damasio, "Deciding advantageously before knowing the advantageous strategy," Science, pp. 1293-1295, 1997.

[3] A. Arad and A. Rubinstein, "Multi-dimensional iterative reasoning in action: The case of the Colonel Blotto game," Journal of Economic Behavior and Organization, vol. 84, pp. 571-585, 2012.

[4] - "The 11-20 money request game: A level-k reasoning study," American Economic Review, vol. 102, pp. 3561-3573, 2012.

[5] F. Lindner and M. Sutter, "Level-k reasoning and time pressure in the 11-20 money request game," Economics Letters, vol. 120, pp. 542-545, 2013.

[6] M. Kocher and M. Sutter, "Time is money: Time pressure, incentives, and the quality of decision-making," Journal of Economic Behavior and Organization, vol. 61, pp. 375-392, 2006.

[7] S. Huck and G. Weizsäcker, "Do players correctly estimate what others do? Evidence of conservatism in beliefs," Journal of Economic Behavior and Organization, vol. 47, pp. 71-85, 2002.

[8] M. Sutter, M. Kocher, and S. Strauss, "Bargaining under time pressure in an experimental ultimatum game," Economics Letters, vol. 81, pp. 341-347, 2003.

[9] T. Strzalecki, "Depth of reasoning and higher order beliefs," Journal of Economic Behavior and Organization, vol. 108, pp. 108-122, 2014.

[10] D. O. Stahl and P. W. Wilson, "On players models of other players: Theory and experimental evidence," Games and Economic Behavior, vol. 10, no. 1, pp. 218-254, 1995.

[11] - "Experimental evidence on players' models of other players," Journal of economic behavior \& organization, vol. 25, no. 3, pp. 309327, 1994.
[12] H. A. Simon, "Rational choice and the structure of the environment." Psychological review, vol. 63, no. 2, p. 129, 1956.

[13] C. Chabris and E. Hearst, "Visualization, pattern recognition, and forward search: Effects of playing speed and sight of the position on grandmaster chess errors," Cognitive Science, vol. 27, pp. 637-648, 2003.

[14] G. Haworth, "Reference fallible endgame play," ICGA Journal, vol. 26, pp. 81-91, 2003.

[15] — "Gentlemen, Stop Your Engines!" ICGA Journal, vol. 30, pp. 150-156, 2007.

[16] H. L. J. V. D. Maas and E.-J. Wagenmakers, "A psychometric analysis of chess expertise," American Journal of Psychology, vol. 118, pp. 29-60, 2005.

[17] M. Guid and I. Bratko, "Computer analysis of world chess champions," ICGA Journal, vol. 29, no. 2, pp. 65-73, 2006.

[18] M. Guid, A. Pérez, and I. Bratko, "How trustworthy is Crafty's analysis of world chess champions?" ICGA Journal, vol. 31, no. 3, pp. 131-144, 2008.

[19] M. Guid and I. Bratko, "Using heuristic-search based engines for estimating human skill at chess," ICGA Journal, vol. 34, no. 2, pp. 71-81, 2011.

[20] K. Regan and G. Haworth, "Intrinsic chess ratings," in Proceedings of AAAI 2011, San Francisco, August 2011.

[21] K. Regan, B. Macieja, and G. Haworth, "Understanding distributions of chess performances," in Proceedings of the 13th ICGA Conference on Advances in Computer Games, Tilburg, Netherlands.

[22] K. Regan and T. Biswas, "Psychometric modeling of decision making via game play," in Proceedings,IEEE Conference on Computational Intelligence in Games, Niagara Falls, Canada, August 2013.

[23] K. Regan, T. Biswas, and J. Zhou, "Human and computer preferences at chess," in Proceedings of the 8th Multidisciplinary Workshop on Advances in Preference Handling (MPref 2014), July 2014.

[24] D. Ferreira, "Determining the strength of chess players based on actual play," ICGA Journal, vol. 35, no. 1, pp. 3-19, March 2012.

[25] - "The impact of search depth on chess playing strength," ICGA Journal, vol. 36, no. 2, pp. 67-80, June 2013.

[26] T. Biswas and K. Regan, "Quantifying depth and complexity of thinking and knowledge," in Proceedings, International Conference on Agents and Artificial Intelligence (ICAART), Lisbon, Portugal, January 2015.

[27] A. Colman, A dictionary of psychology. Oxford New York: Oxford University Press, 2006.

[28] D. Kahneman and A. Tversky, "Prospect theory: An analysis of decision under risk," Econometrica: Journal of the Econometric Society, pp. 263291, 1979

[29] D. Kahneman, "A perspective on judgment and choice: mapping bounded rationality." American psychologist, vol. 58, no. 9, p. 697, 2003.

[30] A. Tversky and D. Kahneman, "Advances in prospect theory: Cumulative representation of uncertainty," Journal of Risk and Uncertainty, vol. 5, pp. 297-323, 1992.

[31] A. Tversky and C. R. Fox, "Weighing risk and uncertainty," Psychological review, vol. 102, no. 2, p. 269, 1995.

[32] D. Kahneman and A. Tversky, "Subjective probability: A judgment of representativeness," in The Concept of Probability in Psychological Experiments. Springer, 1974, pp. 25-48.

[33] - "On the psychology of prediction." Psychological review, vol. 80, no. 4, p. 237, 1973.

[34] _ - "Choices, values, and frames." American psychologist, vol. 39 no. 4, p. $341,1984$.

[35] A. Elo, The Rating of Chessplayers, Past and Present. New York: Arco Pub., 1978.

[36] G. Banks et al., "CCRL rating lists," http://www.computerchess.org.uk/ccrl/, 2013.

[37] V. L. Allis, "Searching for solutions in games and artificial intelligence," $\mathrm{PhD}$ Thesis, 1994.

[38] C. Clark and A. J. Storkey, "Teaching deep convolutional neural networks to play go," CoRR, vol. abs/1412.3409, 2014. [Online]. Available: http://arxiv.org/abs/1412.3409

[39] A. Ban, "Automatic learning of evaluation, with applications to computer chess," Report, 2012.

[40] T. Biswas, "Designing intelligent agents to judge intrinsic quality of human decisions," in Proceedings, International Conference on Agents and Artificial Intelligence (ICAART), Lisbon, Portugal, January 2015. 